\title{
Duality of Photonic Crystal Radiative Structures and Antenna Arrays
}

\author{
Mahdieh Bozorgi and Nosrat Granpayeh* \\ Faculty of Electrical and Computer Engineering, K. N. Toosi University of Technology, \\ Tehran 1431714191, Iran
}

(Received September 29, 2010 : revised November 8, 2010 : accepted November 8, 2010)

\begin{abstract}
In this paper, behaviors of photonic crystal (PC) radiative structures and antenna arrays have been compared for two types of uniform and binomial excitations. Appropriate duality has been shown between them. These results can be generalized to other types of excitation and arrangement of photonic crystal radiative arrays such as linear, planar and circular arrays of three dimensional (3D) photonic crystal termination resonators. Using these results in designing photonic circuits has some advantages for shaping a particular radiative beam at the photonic crystal exit, for instance reducing the divergence angle of the main lobe in order to enhance the directivity, for better coupling, or for splitting the emitted beam, for dividing the output beam to the next devices in photonic integrated circuits (PIC). For analysis and simulation of the photonic crystal structures, the finite difference time domain (FDTD) method has been employed.
\end{abstract}

Keywords: Photonic crystal waveguide, CROW, FDTD, Resonator, Antenna arrays

OCIS codes : (230.0230) Optical devices; (130.3120) Integrated optics devices; (230.1360) Beam splitters; (140.4780) Optical resonators

\section{INTRODUCTION}

The beam shaping mechanisms of optical fields have attracted many researchers from the beginning of the study of electromagnetic fields [1-17]. Recently, it has been shown that beam shaping could be achieved by different kinds of photonic crystal structures due to their photonic band-gap properties [1-13]. One of the methods of beam shaping is engineering the surface modes via corrugations of the surface layer of the photonic crystal waveguide and appropriate termination of the waveguides [11, 18-21]. The defect placement, either point or line defects, at the termination side of the waveguide, modifies the pattern of the radiative beam [22-25]. For these kinds of photonic crystal structures, the mechanism of beam shaping is that the resulting optical field distribution at the photonic crystal exit resembles a multiple point source. The defect types and the arrangement of them, as point sources, cause a particular shape of light emission. The main aim of these studies is designing a particular radiative beam shape at the output of the photonic crystal structures, for example eliminating the side lobes and reducing the divergence angle of the main lobe to increase the directivity for better matching, or splitting the emitted beam for dividing the output light of the photonic crystal device to the next photonic crystal or conventional device in photonic integrated circuits (PICs) [8].

Photonic crystal resonators are special optical components for photonic integrated circuits (PICs). Coupled resonator optical waveguides (CROWs) are constructed with a chain of strongly confined ring resonators [26-29]. The pattern of emitted beam from a CROW surface of a photonic crystal waveguide shows the focusing effect [8].

These remind us of the behavior of the antenna arrays which makes it possible to derive more directional characteristics. The total field of the array is determined by the vector addition of the fields radiated by the individual elements. To provide very directional patterns, it is necessary that the fields from the elements of the array interfere constructively in the desired direction and interfere destructively in the remaining space [30-33].

In this paper, we have used the two dimensional finite difference time domain (FDTD) method to derive the pattern of the photonic crystal radiative defect arrays, and we compare the behavior with that of the antenna arrays.

\footnotetext{
*Corresponding author: granpayeh@eetd.kntu.ac.ir

Color versions of one or more of the figures in this paper are available online.
} 


\section{DETAILS OF STRUCTURES AND SIMULATION SPACE}

We have considered a photonic crystal structure with a square lattice of dielectric rods in air. The relative dielectric constant of the rods is 11.56 , corresponding to the relative dielectric constant of InGaAsP-InP semiconductor at 1.55 $\mu \mathrm{m}$ wavelength [8], and the cross-sectional radius is chosen to be $0.18 a$, where $a$ is the PC lattice constant. For TM polarization with the electric field parallel to the rod axis, the photonic band-gap of the photonic crystal structure is in the normalized frequency $\left(\frac{\omega a}{2 \pi c}\right)$ range of 0.306-0.439, as can be derived by either of the two methods of broadband Gaussian pulse excitation and plane wave expansion (PWE), where $\omega$ is the angular frequency, and $c$ is the velocity of light in free space. All results are presented for TM polarization and have been obtained by the 2D-FDTD method with perfectly matched layer boundary conditions. We have considered $\Delta x=2 r / 10$ as the spatial step in the FDTD method, where $r$ is the cross-sectional radius of the rods. We have chosen radiative defects, by removing some rods at regular intervals at the photonic crystal termination. In this structure each resonator can radiate to free space. The total field of the array is determined by the vector addition of the radiated fields of the individual resonators, the result of which is the radiation pattern of the structure.

In this paper, we have demonstrated the duality of the two structures of radiative photonic crystals, and antenna arrays. But there are some differences. First, the radiative field of a resonator is not analytically known. So, we cannot derive a formula, as in antenna arrays, for total radiative fields of the structure. Second, due to some mutual coupling of the resonators, despite the antenna array, the amplitude relation and the phase differences between the resonators are somehow out of our control. To overcome these two effects, we have used the FDTD method in a large space to calculate the far field, and for the latter we have chosen the distance between the resonators as large as to prevent the mutual coupling. Each resonator is excited by a separate source at the entrance of the waveguide linked to the resonator. Because of the similarity of each resonator excitation, the phase difference can be supposed to be zero. As an example FIG. 1 (a) shows the excitation point, CROW, and the radiative resonator for a single element radiative array, and FIG. 1 (b) shows its pattern, as the unit pattern in an antenna array that is mentioned in Appendix A.

The source is a modulated Gaussian:

$$
S=\cos \left(2 \pi f_{0} t\right) e^{\frac{-4\left(t-T_{0}\right)^{2}}{\sigma^{2}}}
$$

where $f_{0}$ is the resonant frequency of the resonator, $t$ is time, $T_{0}$ is the time of the peak and $\sigma$ is the width controller of the Gaussian pulse.

The normalized resonant frequency of the resonator $\left(\frac{\omega a}{2 \pi c}\right)$ is 0.396 , derived by launching an impulse source (very narrow Gaussian source that its frequency spectrum contains the total band-gap of the photonic crystal) to a photonic crystal waveguide without and with a resonator near it. The resonant frequencies are coupled to the resonator. Thus, the Discrete Fourier Transform (DFT) of the waveguide output at resonant frequency is decreased. So, we can get the resonant frequencies, as shown in FIG. 2.

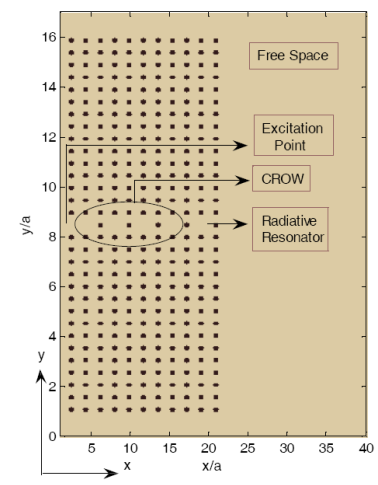

(a)

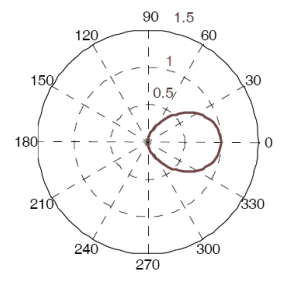

(b)
FIG. 1. (a) Radiative resonator excitation point, CROW and radiative resonator and (b) the polar pattern of the radiative resonator in the azimuthal plane normalized to its peak.

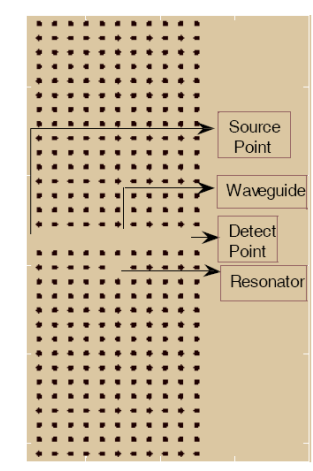

(a)

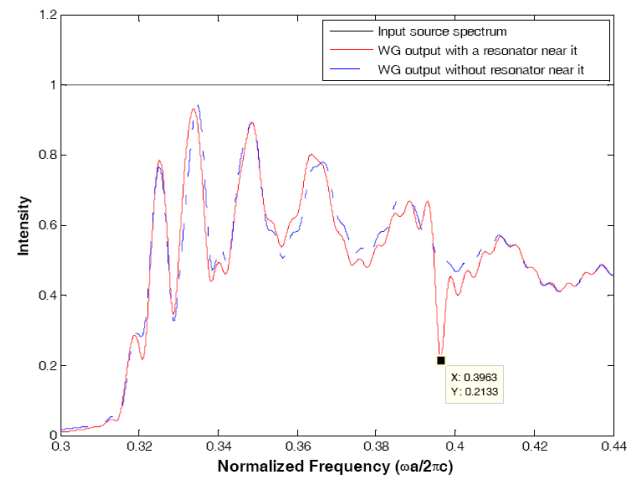

(b)

FIG. 2. (a) The structure for derivation of the resonant frequencies of the resonator and (b) discrete Fourier transform (DFT) of the waveguide input and output, with and without resonator near it. 
In order to restrict the interference of the non-resonant frequencies in free space, we choose $\sigma$ for a narrow spectrum bandwidth, to approach the monochromatic lightwave signal. Moreover, to achieve the more pure resonant frequency in the radiative resonator, we use CROW to link the excited points to the radiative resonator, due to the filtering behavior of CROW.

In this paper, the FDTD software was prepared in $\mathrm{C}^{++}$ and MATLAB and was carried on a Pentium 4 computer with processing capacity of $2.84 \mathrm{GHz}$ and $3.25 \mathrm{~GB}$ of RAM.

\section{THE COMPARISON BETWEEN PHOTONIC CRYSTAL RADIATIVE STRUCTURES AND ANTENNA ARRAYS}

We have considered several photonic crystal radiative arrays by repeating FIG. 1 (a) structure in regular intervals of various multiples of $a$ as arrays element spacing, and arbitrary numbers of array elements. The element distance was chosen such that the coupling between the radiative elements was negligible. As an example, FIG. 3 depicts an array with radiative elements number of $N=3$, and elements distance of $d=6 a$. Then, we excited each of the resonators of the arrays with two types of uniform and binomial excitations. As explained in Appendix A, in the uniform excitation, the incident signals of all CROWs launched to the resonators are the same as Eq. (1), but in the binomial excitation the amplitude of each signal is a multiple of Eq. (1), the multiplication factor of which is chosen from FIG. A. 1. For example in FIG. 3, the excitation of middle CROW is two times greater than that of the bottom and top CROWs. So, as the excitation of each resonator is similar, the phase differences between the elements are supposed to be zero

We have placed some detection points in free space at distances further than $10 \lambda$ say $D=30 a$ from the center of

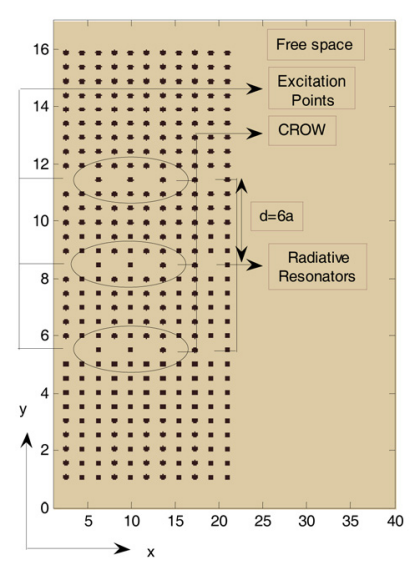

FIG. 3. A photonic crystal radiative array with radiative elements number of $N=3$ and elements distance of $d=6 a$. the array. The detection points are assumed to be at the angle of $-90^{\circ}$ to $90^{\circ}$ with an angle interval of $3^{\circ}$. The amplitude of the electric fields of the detection points gives the total pattern of the arrays.

\subsection{Effects of Variations of The Number of Radiative Elements}

TABLE 1 shows the total pattern for an $N$-element array for various numbers of $N$ and specified element distance $d$, for uniform and binomial excitation. The field pattern formula is the product of the array factor and the unit factor shown in FIG. 1. (b), as those of antenna array given in Appendix A. The same as for antenna arrays in uniform excitation, for a certain $d$, as the array element number $(N)$ is increased, the main lobe direction is constant, but its width becomes narrower, and the number of secondary or minor lobes is increased. These results are in good agreement with the similar case in antenna arrays, given in Appendix A. But there is some exception in these

TABLE 1. The effect of the variation of the number of the radiative elements for two types of uniform and binomial excitations, for a specified elements distance of $d=6 a$.

\begin{tabular}{|c|c|c|}
\hline $\begin{array}{c}\text { Array } \\
\text { elements } \\
\text { number }\end{array}$ & Uniform excitation & Binomial excitation \\
\hline$N=2$ & 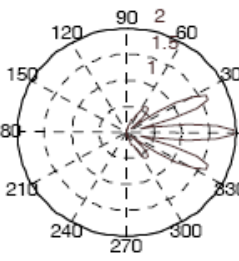 & 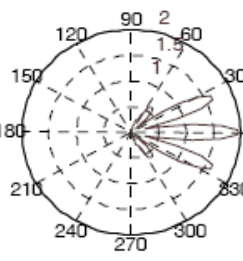 \\
\hline$N=3$ & 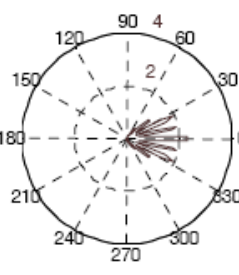 & 180 \\
\hline$N=4$ & 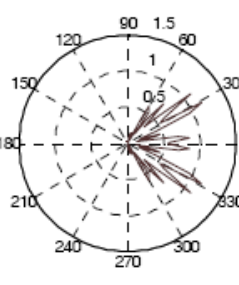 & 180 \\
\hline$N=5$ & 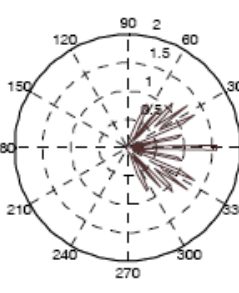 & 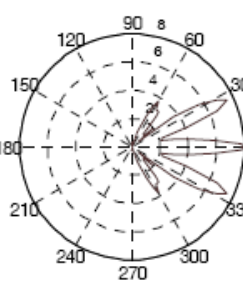 \\
\hline
\end{tabular}




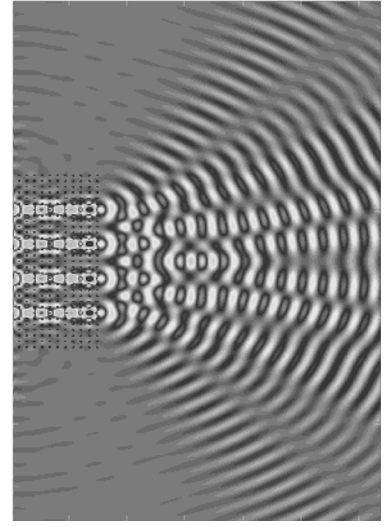

(a)

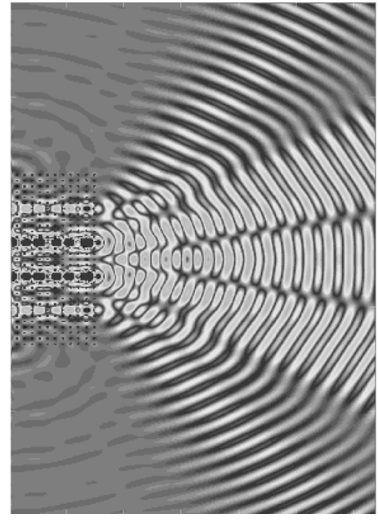

(b)
FIG. 4. The optical field distribution of a 4-element radiative array with elements distance $d=6 a$ for (a) uniform excitation and (b) binomial excitation.

figures such as $N=4$. This is because of the effect of partial coupling between the radiative resonators, variation of the resonant frequency of single resonator and CROW that cause undesirable radiation by non-resonant frequency in free space, and the possibility of changing the phase of each radiative element in CROW path.

In the binomial excitation, as shown in TABLE 1, the same as antenna arrays, given in Appendix A, the minor lobes are eliminated. By increasing $N$, for a certain $d$, in contrast to the uniform case, the number and direction of lobes, do not change, their intensities are increased and hence the directivity is enhanced. But, for the same number of photonic crystal radiative structures, the lobe-widths are more than those of the uniform one. These are again the same as the results for antenna arrays, given in Appendix A. The optical field distributions of a 4-element array with element distance of $d=6 a$ for both excitations of uniform and binomial, are demonstrated in FIG. 4.

\subsection{Effects of Variations of The Elements Distance}

TABLE 2 depicts the patterns of a 3-element array for different distances for uniform and binomial excitations. In uniform excitation, for a given $N$, if the elements distance is increased, the direction of the main lobe is changed, the main lobe becomes narrower, and the number of secondary or minor lobes is increased.

In the binomial excitation, as shown in TABLE 2, for a given $N$, by increasing $d$, the number of lobes is increased, the directions are changed, and the lobe-widths are decreased.

All the results follow the same variations as those of antenna arrays, described in Appendix A. The reasons for some minor exceptions are the mutual coupling between the photonic crystal CROWs, variation of the resonant frequency of single resonator and CROW that cause undesirable radiation by non-resonant frequency in free space, and the possibility of changing the phase of each radiative element in CROW path.
TABLE 2. The effect of the variations of the elements distance for two types of uniform and binomial excitations, for a specified number of radiative elements of $N=3$.

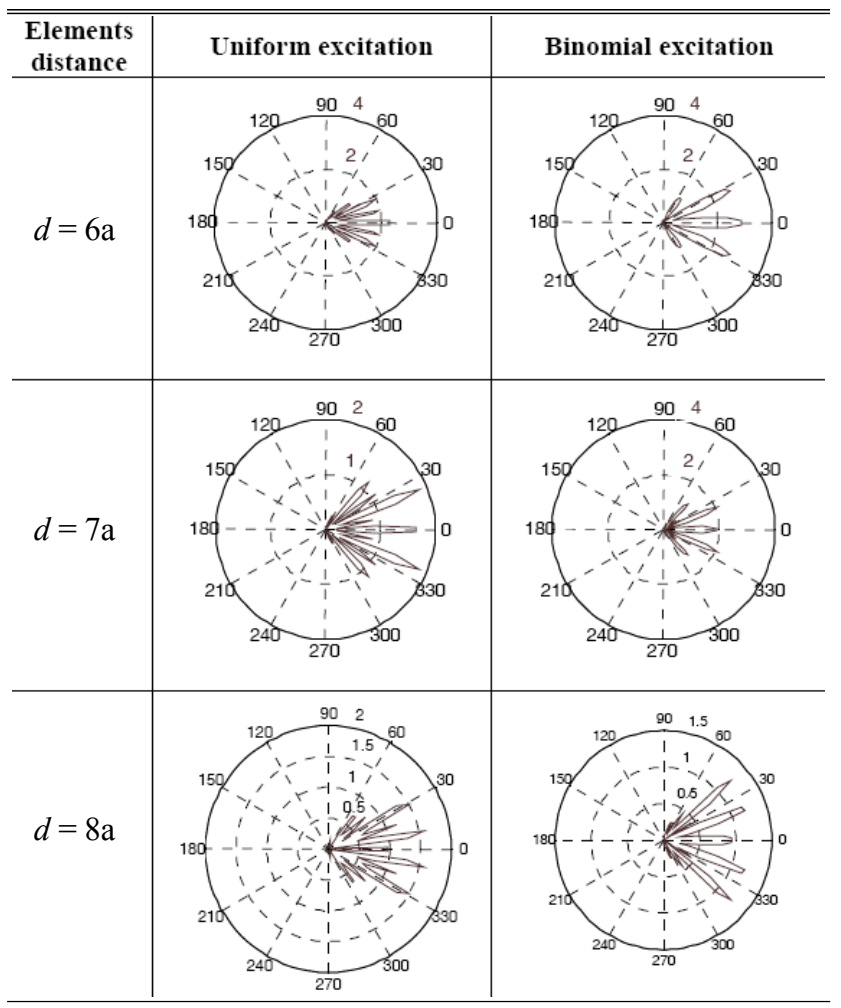

We have analyzed more cases of photonic crystal radiative arrays with various $N$ and $d$ for uniform and binomial excitation and found a considerable duality with antenna arrays. So this duality can be generalized to the other types of excitation such as Dolph-Tshebyscheff arrays, and to the other arrangement of radiative resonators such as any linear, planar and circular arrays of 3D photonic crystal termination resonators.[33] We expect that these structures behave like their equivalent antenna arrays. Therefore, all the experiences in antenna array designs can be employed for manufacturing the photonic crystal beam shaping devices. These devices are very significant in photonic integrated circuits (PICs), due to their capability for enhancing the directivity or splitting the output beams of the photonic crystal devices to be matched to the following devices with single or multiple input ports, respectively, to reduce the connection loss.

\section{CONCLUSION}

In this paper, we have compared the behavior of photonic crystal radiative structures and antenna arrays for two types of uniform and binomial excitations. We found that there is a considerable duality between them. We can generalize the results to the other types of excitations such as Dolph-Tshebyscheff arrays, and to the other arrange- 
ment of radiative resonators such as any linear, planar and circular arrays of 3D photonic crystal termination resonators. Therefore, all the experiences in antenna arrays can be employed in manufacturing the photonic crystal beam shaping devices, in order to reduce the divergence of main lobe to enhance the directivity for better coupling, or splitting the emitted beam in order to divide the output beam to the next devices in photonic integrated circuits (PICs).

\section{APPENDIX A. AN OVERVIEW OF BEAM SHAPING IN ANTENNA ARRAYS}

There are at least five parameters and characteristics for shaping the overall pattern of the antenna arrays. These are the geometrical configuration of the overall array (linear, circular, rectangular, etc.), the relative distances of the elements, the excitation amplitude and phase of the individual elements, and the relative pattern of the individual elements.

The simplest and one of the most practical arrays are formed by placing $N$ antenna elements along a line. For a uniform amplitude and spacing, the total field can be determined by multiplying the array factor (AF) of the isotropic sources by the pattern of the single element, called unit factor (UF). Array factor can be expressed in an alternate, compact and closed form [33]:

$$
A F=\frac{\sin (N \psi / 2)}{\sin (\psi / 2)}
$$

where $\psi=\alpha+\beta d \cos \theta$, where $\alpha$ is the phase difference between the elements, $\beta$ is the propagation constant, $d$ is the elements separation, $\theta$ is the angle of view and $N$ is the number of array elements. Thus the array factor is a function of the number of elements, their geometrical arrangement, their relative phase, and their distance. By varying the number of elements $N$, distance $d$ and/or the relative phase $\alpha$ between the elements the characteristics of the array factor and the total field of the array can be controlled.

For $\psi=2 k \pi / N, k=1,2,3, \cdots$ the array factor has nulls, and for $\psi=2 k \pi, k=0,1,2,3, \cdots$. Eq. (A.1) attains its maximum values. Thus the number of nulls that can exist will be a function of the number of elements $N$, element distance $d$ and the phase excitation difference $\alpha$. Here, we suppose $\alpha$ to be zero and consider the effects of the variations of the other parameters. Thus, for uniform excitation, for a certain $d$, as the number of array elements $(N)$ is increased:

- The direction of the main lobe does not change,

- The main lobe becomes narrower,

- The number of secondary or minor lobes is increased.

Also, for a given $N$, if the elements distance $d$ is increased:
- The direction of the main lobe is changed,

- The main lobe becomes narrower,

- The number of secondary or minor lobes is increased.

Another case is the uniform spacing and non-uniform amplitude, an example of which is binomial arrays. The excitation coefficients of the binomial arrays for different values of $N$ are given in FIG. A.1 [33].

For example in a three element array that has the current ratio of 1:2:1, we can decompose this array into two 2-element uniform arrays, as shown in FIG. A.2, with element distance $d$.

So the array factor will be:

$$
\mathrm{AF}=[2 \cos (\psi / 2)][2 \cos (\psi / 2)]=[2 \cos (\psi / 2)]^{(2)}
$$

Thus, a general array factor formula for $N$ elements array will be:

$$
\mathrm{AF}=[2 \cos (\psi / 2)]^{(N-1)}
$$

The array factor of 2-element uniform antenna array does not have minor lobes, thus, the binomial arrays will also be the same. By increasing $N$, for a certain $d$, despite the uniform case:

- The number and direction of the lobes are not changed,

- The lobe intensities are increased,

- Hence, the directivity is enhanced,

- For the same N, its lobe-width is more than that of the uniform one.

For a given $N$, by increasing $d$ :

- The number of lobes is increased,

- The directions are changed,

- The lobe-width is decreased [33-34].

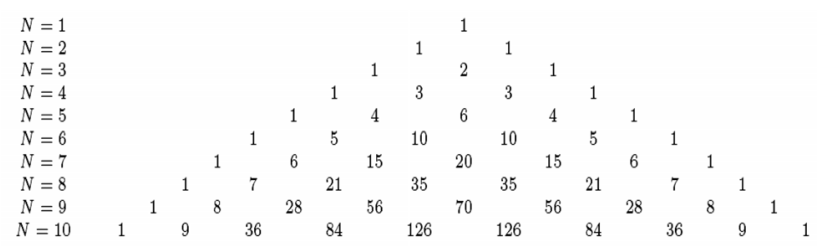

FIG. A.1. The excitation coefficients of the binomial arrays for different numbers of array elements [33].

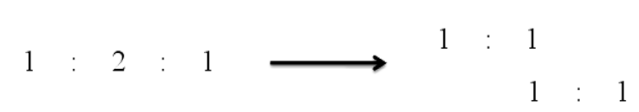

FIG. A.2. Decomposition of the three element binomial array into two 2-element uniform arrays. 


\section{ACKNOWLEDGMENT}

The authors would like to thank Education and Research Institute for ICT (ERICT), (formerly, Iran Telecommunication Research Center (ITRC)) for the financial support of this project.

\section{REFERENCES}

1. H. A. Bethe, "Theory of diffraction by small holes," Phys. Rev. 66, 163-182 (1944).

2. E. Yablonovitch, "Inhibited spontaneous emission in solid state physics and electronics," Phys. Rev. Lett. 58, 2059-2062 (1987).

3. S. John, "Strong localization of photonics in certain disordered dielectric super-lattices," Phys. Rev. Lett. 58, 2486-2489 (1987).

4. O. Painter, R. K. Lee, A. Scherer, A. Yariv, J. D. O'Brien, P. D. Dapkus, and I. Kim, "Two-dimensional photonic band-gap defect mode laser," Science 284, 1819-1821 (1999).

5. J. D. Joannopoulos, P. R. Villeneuve, and S. Fan, "Photonic crystals: putting a new twist on light," Nature 386, 143-149 (1997).

6. K. M. Leung and Y. F. Liu, "Full vector wave calculation of photonic band structure in face-centered-cubic dielectric media," Phys. Rev. Lett. 65, 2646-2649 (1990).

7. G. Guida, A. Delustrac, and A. Priou, "An introduction to photonic band-gap (PBG) materials," PIER 41, 1-20 (2003).

8. Z.-H. Zho, W.-M. Ye, J.-R. Ji, X.-D. Yuan, and C. Zen, "Enhanced transmission and directional emission via coupled resonator optical waveguides," Appl. Phys. B 86, 327-331 (2007).

9. L. Pajewski, L. Rinaldi, and G. Schettini, "Enhancement of directivity using 2D electromagnetic crystals near the band-gap edge: a full-wave approach," PIER 80, 179-196 (2008)

10. W. Y. Liang, J. W. Dong, and H. Z. Wang, "Directional emitter and beam splitter based on self collimation effect," Opt. Express 15, 1234-1239 (2007).

11. H. Kurt, "Theoretical study of directional emission enhancement from photonic crystal waveguides with tapered exits," IEEE Photonics Technol. Lett. 20, 1682-1684 (2008).

12. K. B. Chung, "Properties of surface modes used for directional emission from photonic crystal waveguides," J. Opt. Soc. Korea 12, 7-12 (2008).

13. K. B. Chung, "Effects of surface termination on directional emission from photonic crystal waveguides," J. Opt. Soc. Korea 12, 13-18 (2008).

14. S. Kim, Y. Lim, J. Park, and B. Lee, "Bundle beaming from multiple subwavelength slits surrounded by dielectric surface gratings," IEEE J. Lightwave Technol. 28, 2023-2029 (2010).

15. B. Lee, S. Kim, H. Kim, and Y. Lim, "The use of plasmonics in light beaming and focusing," Prog. in Quantum Electron. 34, 47-87 (2010).

16. H. Kim, J. Park, and B. Lee, "Tunable directional beaming from subwavelength metal slits with metal-dielectric composite surface gratings," Opt. Lett. 34, 2569-2571 (2009).
17. S. Kim, H. Kim, Y. Lim, and B. Lee, "Off-axis directional beaming of optical field diffracted by a single subwavelength metal slit with asymmetric dielectric surface gratings," Appl. Phys. Lett. 90, 051113 (2007).

18. P. Kramper, M. Agio, C. M. Soukoulis, A. Birner, F. Muller, R. B. Wehrspohn, U. Gosele, and V. Sandoghar, "Highly directional emission from photonic crystal waveguides of sub-wavelength width," Phys. Rev. Lett. 92, 113903 (2004).

19. E. Moreno, F. J. Garcia, and L. Martin-Moreno, "Enhanced transmission and beaming of light via photonic crystal surface modes," Phys. Rev. B 69, $121402-121404$ (2004).

20. S. K. Morrison and Y. S. Kivshar, "Engineering of directional emission from photonic crystal waveguides," Appl. Phys. Lett. 86, 081110 (2005).

21. I. Bulu, H. Caglayan, and E. Ozbay, "Beaming of light and enhanced transmission via surface modes of photonic crystals," Opt. Lett. 30, 3078-3080 (2005).

22. C. C. Chen, T. Pertsch, R. Iliev, F. Lederer, and A. Tunnermann, "Directional emission from photonic crystal waveguides," Opt. Express 14, 2423-2428 (2006).

23. K. Guven and E. Ozbay, "Directivity enhancement and deflection of the beam emitted from a photonic crystal waveguide via defect coupling," Opt. Express 15, 14973-14978 (2007).

24. Y. Zhang, Y. Zhang, and B. Li, "Highly efficient directional emission from photonic crystal waveguides for coupling of freely propagated terahertz waves into Si slab waveguides," Opt. Express 15, 9281-9286 (2007).

25. Z. Li, K. Aydin, and E. Ozbay, "Highly directional emission for photonic crystals with a wide bandwidth," Appl. Phys. Lett. 91, 121105 (2007).

26. A. Yariv, Y. Xu, R. K. Lee, and A. Scherer, "Coupled resonator optical waveguide: a proposal and analysis," Opt. Lett. 24, 711-713 (1999).

27. S. Oliver, C. Smith, M. Rattier, H. Benisty, C. Weisbuch, T. Krauss, R. Houdre, and U. Oesterle, "Mini-band transmission in a photonic crystal coupled resonator optical waveguide," Opt. Lett. 26, 1019-1021 (2001).

28. M. Bayindir, B. Temelkuran, and E. Ozbay, "Tight binding description of the coupled defect modes in three dimensional photonic crystals," Phys. Rev. Lett. 84, 2140-2143 (2000).

29. T. D. Happ, M. Kamp, A. Forchel, J. Gentner, and L. Goldstein, "Two dimensional photonic crystal coupled defect laser diode," Appl. Phys. Lett. 82, 4-6 (2003).

30. F. Ares, G. Franceschetti, and J. A. Rodriguez, "A simple alternative for beam reconfiguration of array antennas," PIER 88, 227-240 (2008).

31. H. J. Zhou, B. H. Sun, J. F. Li, and Q. Z. Lin, "Efficient optimization and realization of a shaped beam planar array for very large array application," PIER 86, 1-10 (2009).

32. S. W. Yang, Y. K. Chen, and Z. P. Nie, "Simulation of time modulated linear antenna arrays using the FDTD method," PIER 98, 175-190 (2009).

33. C. A. Balanis, Antenna Theory: Analysis and Design (Wiley-Interscience, New Jersey, USA, 2005).

34. E. C. Jordan and K. G. Balmain, Electromagnetic Waves and Radiating Systems (Prentice-Hall, New Jersey, USA, 1968). 